\title{
Preparation and Properties of 3D Chitosan Microtubes
}

\author{
Natalia O. Gegel, ${ }^{1}$ Anna B. Shipovskaya, ${ }^{1,2}$ Luba S. Vdovykh, ${ }^{2}$ and Tatiana S. Babicheva ${ }^{2}$ \\ ${ }^{1}$ Educational and Research Institute Nanostructures and Biosystems, Saratov State University, 83 Ulitsa Astrakhanskaya, \\ Saratov 410012, Russia \\ ${ }^{2}$ Institute of Chemistry, Saratov State University, 83 Ulitsa Astrakhanskaya, Saratov 410012, Russia \\ Correspondence should be addressed to Natalia O. Gegel; gegelno@yandex.ru
}

Received 24 April 2014; Revised 15 July 2014; Accepted 17 July 2014; Published 12 August 2014

Academic Editor: Jie Han

Copyright (C) 2014 Natalia O. Gegel et al. This is an open access article distributed under the Creative Commons Attribution License, which permits unrestricted use, distribution, and reproduction in any medium, provided the original work is properly cited.

The preparation of 3D chitosan microtubes from polymer solutions in citric and lactic acids by the wet and dry molding methods is described. The mechanism of formation of the insoluble polymeric layer constructing the walls of these microtubes is characterized. The microtubes obtained from chitosan solutions in citric acid are found to have a fragile porous inner layer. For those obtained from chitosan solutions in lactic acid the morphology, elastic-deformation properties, physicomechanical properties, and biocompatibility were assessed. These samples have smooth outer and inner surfaces with no visible defects and high values of elongation at break. The strength of the microtubes obtained by the dry method is much higher than in the case of the wet one. A high adhesion and high proliferative activity of the epithelial-like MA-104 cellular culture on the surface of our microtubular substrates in model in vitro experiments were revealed. Prospects of using chitosan microtubes as vascular prostheses are suggested.

\section{Introduction}

Now, commercial vascular prostheses made of synthetic nonbiodegradable polymers, such as polytetrafluoroethylene and polyethylene terephthalate [1,2], or made of biological tissues, for example, animal xenopericard [3], are commonly used to replace vascular defects. However, these prostheses are far from ideal and have a number of disadvantages; in particular, they do not biodegrade in a natural metabolic route and, hence, cannot be used for short-term stay in the body. On the contrary, the use of prostheses made of biodegradable polymers would enable one to avoid re-remodeling of vessels, which would be especially important in the case of prostheses for children whose bodies undergo physiological maturation. For example, prostheses made of biodegradable polymers eventually grow their own connective tissue and human endothelial and, concurrently, biodegrade by natural metabolism and, consequently, are excreted from the body. As a result, a new living organ, a vessel, is formed and grows with the patient. When prostheses used are made of nonbiodegradable polymers, no vessel growth with the patient's maturation occurs. Over time, therefore, such an implant must be replaced by a larger vascular prosthesis, which entails a new surgery. A successful surgery carried out in the Russian Federation of implantation of a vascular prosthesis made of a biodegradable material (obtained from Euroderm $\mathrm{GmbH}$ ) to a 12 -year-old child with a congenital heart disease is reported in [4]. Full biodegradation of the polymer matrix of the prosthesis and the formation of a new vessel are expected to occur in 9 months.

To expand the range of such implants, it is urgent to search for new polymers and methods for making vascular prostheses biodegradable in the body by natural metabolism with the simultaneous restoration of the cellular composition of the vascular wall in vivo.

Approaches are known to the preparation of vascular tissue-engineered grafts by electrospinning as hollow cylindrical structures made of synthetic biodegradable polymers: poly( $\varepsilon$-caprolactone) [5], elastic crosslinked urethane-doped polyester [6], aligned poly(L-lactide-co-epsilon-caprolactone) (75/25) [7] or poly(L-lactide-co-glycolide) [8], a blend of polydioxanone and soluble elastin [9], compositions based on poly(L-lactide-co-glycolide) and poly( $\varepsilon$-caprolactone) $[10,11]$. Hu et al. [12] reported the preparation of a kind of blood vessel structure with a microtubular orientation to mimic the natural structure, from solutions of poly(lactideco-glycolide) $(70 / 30)$ in 1,4-dioxane by an improved 
heat-induced phase separation. A kind of microtubular orientation-structured blood vessel mimicking the natural structure was fabricated with poly(lactide-co-glycolide) $(70 / 30)$ (PLGA (70/30)) solutions in 1,4-dioxane by an improved thermal-induced phase separation (TIPS) technique. The vascular grafts developed are promising in repairing damaged blood vessels, nerves, and so forth. The formation of a living organ on a polymer matrix occurs due to the fact that the body's stem cells migrate into the zone of damage where they actively divide to promote tissue repair. By the time of the final formation of the organ, the matrix material must be completely removed from the body. For example, poly( $\varepsilon$-caprolactone $)$ is known to be destructed in the body during more than one year [5]. It is believed that this period would be sufficient to create a comprehensive living blood vessel.

To reduce thrombus and to shorten the degradation period of the prosthetic polymer matrix to form a small-diameter living blood vessel, a two-phase tubular scaffold made of poly(diol citrate), a new-class biodegradable polyester elastomer, is proposed to be used [13].

3D blood vessel analogs are also fabricated using heteropolysaccharides, namely, alginate, gellan gum, K-carrageenan, and hyaluronan $[14,15]$. One of perspective polymers to create biodegradable prostheses is chitosan $(\mathrm{CH})[16-$ 18]. This aminopolysaccharide is biocompatible with human tissues, is nontoxic, is nonallergenic, and promotes tissue regeneration. In the process of preparing chitosan, its antigenicity is almost completely lost. As the melting point of chitosan is significantly higher than its thermal decomposition temperature, all materials, in particular microtubes, are prepared from its solutions.

The literature describes several formation methods and properties of chitosan microtubes (hollow cylindrical structures) obtained by electrospinning [19], multistage electroforming with other polymers used [20], molding in special molds [21, 22], application of chitosan onto a preprepared polymer woven base [23], or a combination of several different approaches, for example, electroforming and wire molding, followed by treatment in a precipitation bath [24]. All these methods are multistage and complicated. To enhance the stability, strength, and other properties, the microtubes obtained are further subjected to various treatments, in particular, keeping in ethyl alcohol [20], cooled toluene [23], aqueous ammonia and caustic soda [24], and so forth. A mixture of trifluoroacetic acid and methylene chloride is also used for the preparation of chitosan solutions [19, 24], which certainly would adversely affect the biocompatibility and other biochemical properties of the final material.

Earlier, we demonstrated the possibility of obtaining microtubes from water-acidic chitosan solutions in a relatively simple manner of wet spinning to a precipitation bath $[25,26]$. The present work describes the formation and properties of chitosan microtubes obtained from a solution of this aminopolysaccharide in organic acids of pharmacopeial destination by both wet and dry molding methods. To form a water-insoluble polymer layer constituting the wall of our microtubes, the reaction of conversion of protonated polysalt to polybase was used.

\section{Material and Methods}

2.1. The Reagents Used. Chitosan with a molecular weight of $700 \mathrm{kDa}$ and a deacetylation degree of $80 \mathrm{~mol}$.\% was received from Bioprogress Ltd. (Russian Federation). Pharmacopeic acids, namely, citric acid $C_{\mathrm{CA}}=5 \%$ and lactic acid $C_{\mathrm{LA}}=$ $1.5 \%$, were used as organic solvents. Chitosan solutions with $C_{\mathrm{CH}}=2.5-5.0 \mathrm{wt}$.\% were prepared by dissolving a sample of air-dried powder of the polymer in the presence of the corresponding acid on a magnetic stirrer at room temperature during $5-7 \mathrm{~h}$. The resulting solution was left to remove air bubbles for $24 \mathrm{~h}$ and used to form microtubes by both wet and dry methods. To transfer chitosan in a microtube from its salt form to the water-insoluble polybasic one, a $5 \% \mathrm{NaOH}$ or $50 \% \mathrm{~N}\left(\mathrm{C}_{2} \mathrm{H}_{4} \mathrm{OH}\right)_{3}$ solution was used. To prepare solutions of the acids, alkalis, and triethanolamine, bidistilled water was taken. Xenopericardial biomaterial was gotten from bovine pericardium, the manufacturer Cardioplant Ltd. (Russian Federation).

2.2. Technique to Obtain of Microtubules. Figure 1 presents a diagram of microtube preparation from chitosan solutions in citric and lactic acids by the wet and dry molding methods. The microtube formation process was performed as follows. A glass rod with a circular cross-section (the diameter was $5 \mathrm{~mm}$ ) was vertically immersed into a chitosan solution and kept for $1 \mathrm{~min}$ for its surface to be coated with a uniform layer of the forming solution. When using the wet method, the rod with the deposited layer of the forming polymer solution in an organic acid was kept in a $\mathrm{NaOH}$ solution for $12 \mathrm{~h}$. During this time, the reaction of conversion of the protonated chitosan polysalt into polybase proceeded and a water-insoluble polymer layer was thus formed to construct the microtube's wall as a hollow cylinder. In the dry method, the rod with the chitosan solution layer was immersed into a $\mathrm{N}\left(\mathrm{C}_{2} \mathrm{H}_{4} \mathrm{OH}\right)_{3}$ solution for $1 \mathrm{~min}$, removed, and dried in an oven at $45-50^{\circ} \mathrm{C}$ until complete solvent evaporation (4$5 \mathrm{~h}$ ). Then it was immersed in an aqueous $\mathrm{NaOH}$ solution for $20 \mathrm{~min}$ for the polysalt-polybase reaction to form the microtube's walls. Samples of the microtubes obtained by both wet and dry methods were removed from the rod, washed with distilled water until neutral, and stored in the swollen state in distilled water.

2.3. Physicochemical Methods. The morphology of sample chitosan microtubes was evaluated both visually and on a MIRA $\backslash \backslash L M U$ SEM (Tescan, Czech Republic) with the following parameters: color, transparency, and the uniformity of the inner and outer surfaces. The wall thickness $(d$, $\mathrm{mm}$ ) of microtubes was measured with an electronic digital outside micrometer CT 200-521 (China) with a scale division of $10 \mu \mathrm{m}$. Measurements were carried out several times at different sites of every microtube and then the average thickness was calculated. Elastoplastic properties were determined on a tensile testing machine of uniaxial tension TiraTest 28005 (Germany) with a load cell of $50 \mathrm{~N}$. Microtubes were cut along, unfolded, and fixed in the terminals of the tensile testing machine as plates. Every sample was tested at least 5 times. The breaking force and elongation at break 


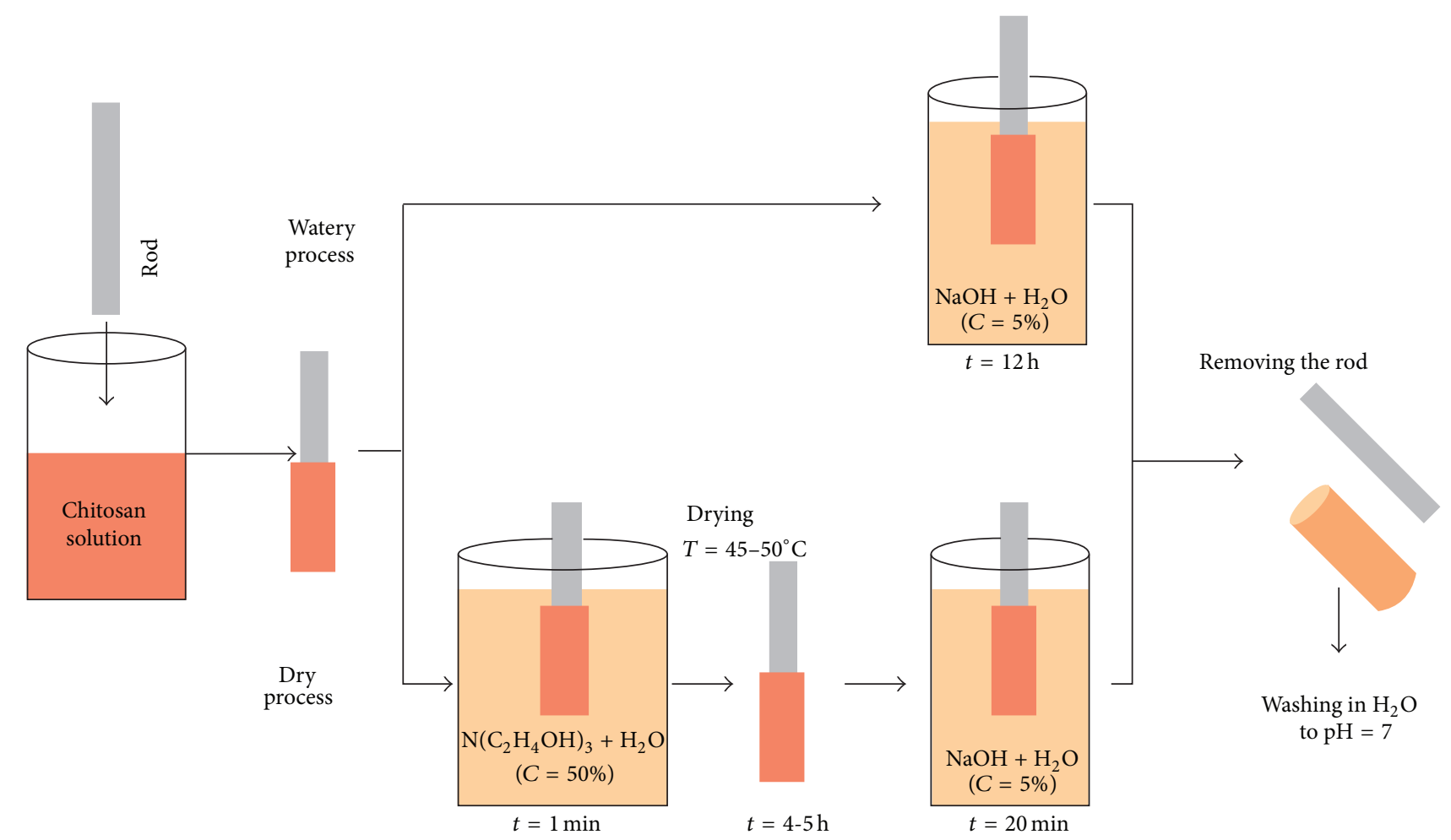

FIGURE 1: A scheme of making microtubes from chitosan solutions in lactic and citric acids by the wet and dry molding methods.

were calculated according to standard procedures. The data obtained were plotted as stress-strain curves $\sigma=f(\varepsilon)$. The tensile stress $(\sigma, \mathrm{MPa})$ was calculated from the crosssectional area of the original sample. The elongation at break $(\varepsilon, \%)$ was calculated using the original length of the sample microtube.

2.4. Biochemical Methods. To assess the biocompatibility of our microtubes, the MA-104 culture of epithelial-like cells (a rhesus monkey's embryonic kidney) was used. Cells were cultivated under sterile conditions: in a laboratory specially equipped with a complex of "clean" laminates (second class protection, NuAire, USA). A microtube sample was sterilized in a $70 \%$ ethanol solution for $20 \mathrm{~min}$, placed into sterile Petri dishes (FalconBD), and filled with the DMEM culture medium (Biolot, RF) supplemented with $10 \%$ fetal bovine serum (HyClone, UK) and an antibiotic-antimycotic mixture, and a suspension of the cell culture was introduced at a concentration of $1 \cdot 10^{4}$ cells $/ \mathrm{cm}^{3}$. Culturing was performed in a SanyoMCO-18 $\mathrm{M} \mathrm{CO}_{2}$ incubator (Sanyo, Japan) in a $5 \% \mathrm{CO}_{2}$ atmosphere at $37^{\circ} \mathrm{C}$. The culture medium was replaced on changing the medium indicator color. Cells were cultivated during 7 days. The adhesion and proliferation of the cell culture on our microtubes were evaluated daily on an inverted IIB-P microscope (Russian Federation). Because the polymeric substrates under study had the shape of a hollow cylinder with a nonplanar surface, the approach described in $[27,28]$ was used to evaluate the cultured cell morphology. To this purpose, the samples with the cell culture grown on their surface were washed with PBS phosphate buffer (Biolot,
Russian Federation), covered with a $5 \%$ formalin solution, and dried at $23 \pm 2^{\circ} \mathrm{C}$ for $24 \mathrm{~h}$. A layer of gold was deposited on the obtained sample $5 \mathrm{~nm}$ thick on a device K450X Carbon Coater (Deutschland). SEM photos were obtained on a MIRA \\LMU scanning electron microscope (Tescan, Czech Republic) at operating with a $30 \mathrm{kV}$ accelerating voltage, 300 pA carrying a current. Observation of adhesion and cell proliferation was carried out and obtained with an inverted "Biolam P" microscope (Russian Federation) with a digital CCD camera DMS $300 \ll$ Scopotek» (China): 10x lens, 3 Mpx resolution.

\section{Results}

The morphology of the outer and inner surfaces of our molded microtube is shown in Figure 2. A microtube obtained from a chitosan solution in citric acid by the wet process is opaque and has a brittle porous inner layer (Figures 2(a) and 2(c)). The microtube samples obtained from a chitosan solution in lactic acid by either wet or dry method have smooth inner and outer surfaces, with no visible defects (Figures 2(c)-2(f)). These significant differences in the morphology of the samples obtained from chitosan solutions in different acids may be due to some physicochemical properties of these carboxylic acids (citric and lactic acids being tribasic and monobasic, resp.), probably affecting the kinetics of the chemical polysalt-polybase reaction and phase separation.

Due to the excessive porosity of the inner layer of microtubes obtained from chitosan solutions in citric acid, 


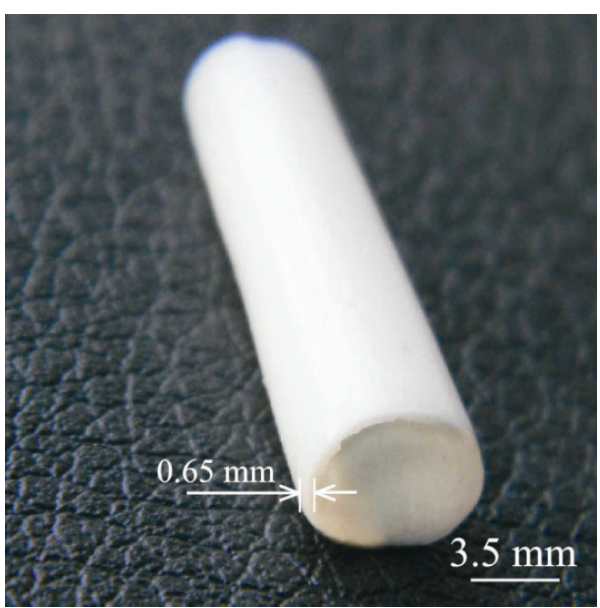

(a)

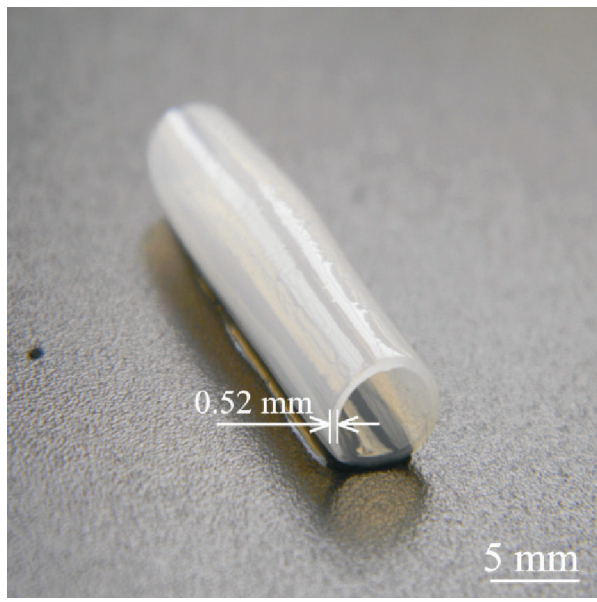

(c)

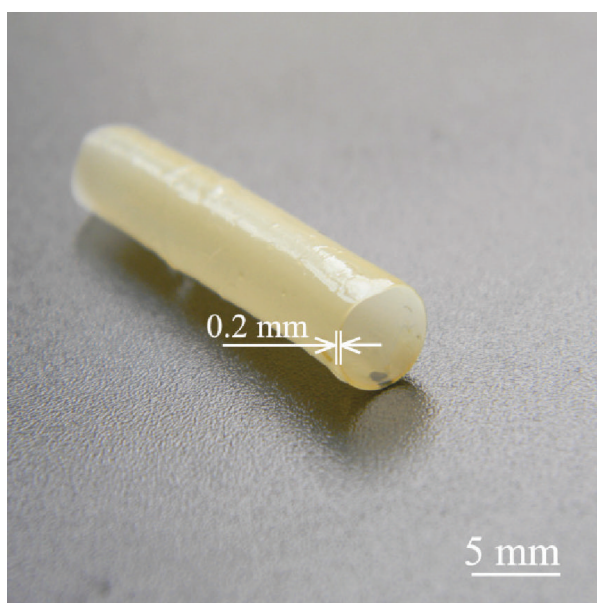

(e)

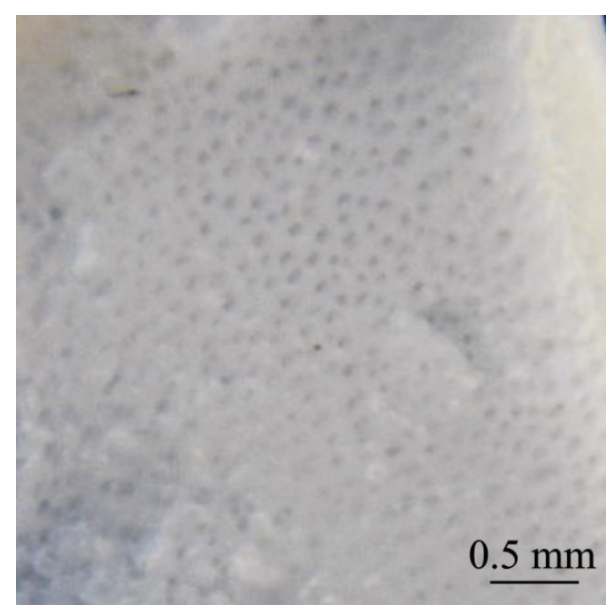

(b)

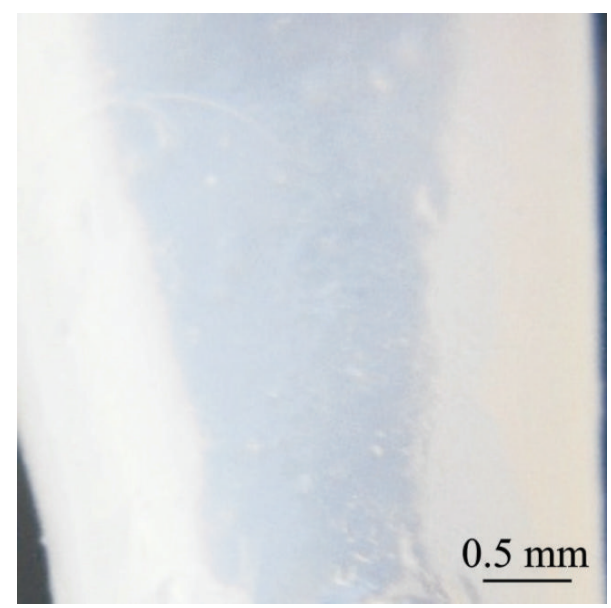

(d)

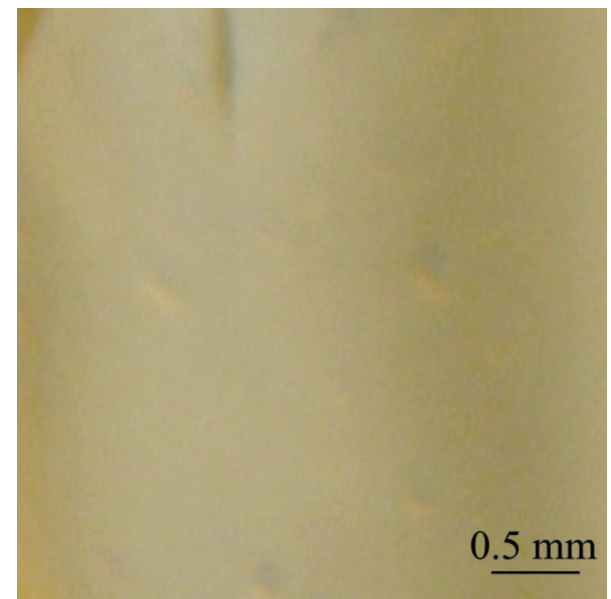

(f)

Figure 2: Photos of the external ((a), (c), (e)) and internal ((b), (d), (f)) surfaces of microtubes made from the chitosan solution with $C_{\mathrm{CH}}=$ 5.0 wt. \% in citric acid ((a), (b)) and with $C_{\mathrm{CH}}=3.0 \mathrm{wt} . \%$ in lactic acid ((c)-(f)) by the wet $((\mathrm{a})-(\mathrm{d}))$ and dry methods $((\mathrm{e}),(\mathrm{f}))$.

microtube samples obtained from chitosan solutions in lactic acid were chosen for further tests.

As mechanical properties are very important to predict the behavior of a material in a biological medium, the elastic-plastic properties of our microtubes formed from chitosan solutions in lactic acid by the wet and dry methods were evaluated (Table 1, Figure 3).

The biocompatibility of chitosan microtubes was assessed on the example of the culture MA-104 of epithelial-like cells in model experiments in vitro (Figure 4). 
TABle 1: Physicomechanical properties of microtube samples prepared from chitosan solutions in 1.5\% lactic acid.

\begin{tabular}{|c|c|c|c|c|}
\hline $\begin{array}{l}\text { Chitosan concentration } \\
\left(C_{\text {chit }}\right), \text { wt. } \% \\
\end{array}$ & Way of formation & $\begin{array}{l}\text { Microtube wall thickness } \\
(d), \mathrm{mm}\end{array}$ & $\begin{array}{l}\text { Relative elongation } \\
(\varepsilon), \%\end{array}$ & $\begin{array}{c}\text { Elongation at break } \\
(\sigma), \mathrm{MPa}\end{array}$ \\
\hline 2.5 & \multirow{5}{*}{ Wet } & $0.50 \pm 0.06$ & $27.0 \pm 4.20$ & $0.10 \pm 0.03$ \\
\hline 2.75 & & $0.41 \pm 0.02$ & $30.0 \pm 3.50$ & $0.22 \pm 0.04$ \\
\hline 3.0 & & $0.52 \pm 0.04$ & $34.0 \pm 2.15$ & $0.70 \pm 0.15$ \\
\hline 3.25 & & $0.40 \pm 0.02$ & $45.0 \pm 2.50$ & $0.30 \pm 0.01$ \\
\hline 3.5 & & $0.54 \pm 0.04$ & $23.0 \pm 5.15$ & $0.25 \pm 0.04$ \\
\hline 2.75 & \multirow{4}{*}{ Dry } & $0.29 \pm 0.02$ & $8.0 \pm 0.5$ & $8.0 \pm 0.8$ \\
\hline 3.0 & & $0.20 \pm 0.01$ & $21 \pm 1.2$ & $12.0 \pm 0.3$ \\
\hline 3.25 & & $0.35 \pm 0.05$ & $27 \pm 1.2$ & $5.5 \pm 0.3$ \\
\hline 3.5 & & $0.24 \pm 0.01$ & $38.5 \pm 0.3$ & $4.2 \pm 0.1$ \\
\hline
\end{tabular}

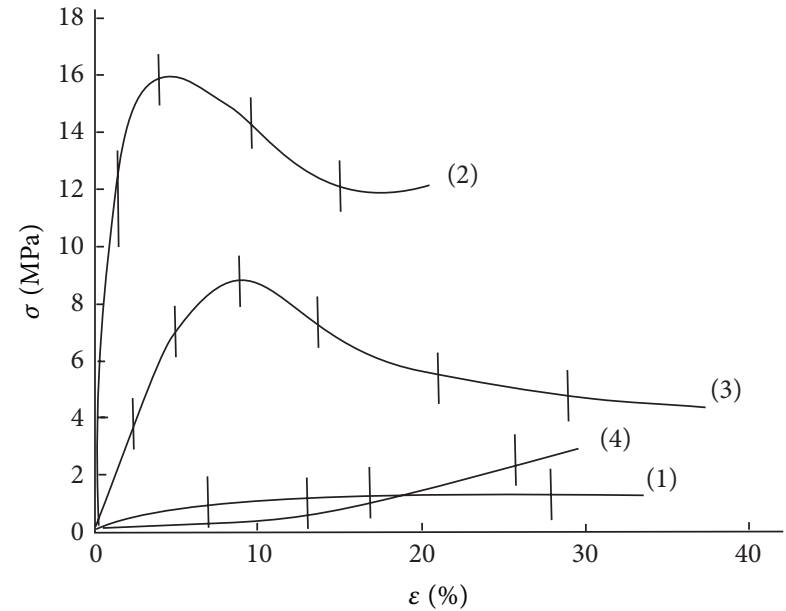

FIGURE 3: Stress-strain curves for chitosan microtube samples (13 ) and xenopericard samples (4). The microtube samples obtained from chitosan solutions with $C_{\text {chit }}=3.0(1,2)$ and $3.5 \mathrm{wt} . \%(3)$ by the wet (1) and dry methods $(2,3)$.

\section{Discussion}

During the formation of the chitosan microtube's walls by the wet process, the protonated polysalt-polybase reaction and phase separation processes occurred simultaneously: first, on the border of two liquid phases and then on the solid-liquid interphase. When forming the walls of microtubes by the dry method, the polysalt-polybase conversion proceeded in a solid (condensed) polymer phase. The mechanism of wall formation of microtubes during both dry and wet molding methods is as follows (Figure 5).

In the initial water-acidic solution, due to the side $\mathrm{NH}_{2}$ group protonation, chitosan is polysaltic $\left(\sim-\mathrm{NH}_{3}{ }^{+}\right)$. In an alkaline medium, the saltic groups are converted into amine ones to form a polybase $\left(\sim-\mathrm{NH}_{2}\right)$, and the solubility of the polymer is lost $(\downarrow)$. A scheme of these transformations is as follows:

$$
\begin{gathered}
\sim-\mathrm{NH}_{2}+\mathrm{HAn} \longrightarrow \sim-\mathrm{NH}_{3}{ }^{+}+\mathrm{An}^{-} \\
\sim-\mathrm{NH}_{3}{ }^{+}+\mathrm{OH}^{-} \longrightarrow \sim-\mathrm{NH}_{2}(\downarrow)+\mathrm{H}_{2} \mathrm{O}
\end{gathered}
$$

In the wet molding method, when a glass rod (its crosssectional view is shown in Figure 5 as a black circle) coated with a chitosan solution layer is immersed into an alkaline solution, the protonated $-\mathrm{NH}_{3}{ }^{+}$groups begin to interact with $\mathrm{OH}^{-}$ions at the interface between the two liquid phases (in Figure 5(a) the interface is marked in dashed lines). Some part of the macromolecular chitosan chains or their fragments pass from the saltic form to the polybasic one, thereby forming an initial polymer layer insoluble in both water-acidic and water-basic media (shown in Figure 5(b) as a circular ring of a width $h_{1}$ ). This layer is a semipermeable membrane, that is, permeable for $\mathrm{H}_{2} \mathrm{O}$ molecules, $\mathrm{Na}^{+}$, $\mathrm{OH}^{-}$ions and impermeable to chitosan macromolecules. This leads to the fact that a concentration gradient arises at the boundary between the semipermeable membrane and the chitosan solution, whereby $\mathrm{OH}^{-}$ions still remain to diffuse into the chitosan solution and to react with its $-\mathrm{NH}_{3}{ }^{+}$groups to form another layer of the semipermeable membrane (Figure 5(c), an annular ring of a width $h_{2}$ ). Then the diffusion processes of $\mathrm{OH}^{-}$ions and their interaction with $-\mathrm{NH}_{3}{ }^{+}$groups continue until complete exhaustion of the saltic groups (Figure 5(d), an annular ring of width $h_{3}$ ). As a result of these processes, the wall of the microtube is formed. The resulting microtube sample can be easily removed from its glass rod. Similar kinetics of phase separation during the polysalt-polybase processes was observed when preparing $3 \mathrm{D}$ chitosan rods [29].

In the case of the dry method, the polysalt-polybase conversion reaction proceeds inside the bulk polymer and is caused solely by the diffusion of low-molecular ions $\left(\mathrm{OH}^{-}, \mathrm{Na}^{+}\right)$into the condensed layer of the polymer due to the concentration gradient at the $\sim-\mathrm{NH}_{3}{ }^{+} \mid \mathrm{NaOH}$ interface, directed to leveling the chemical potential of the low-molecular-weight component in the system.

According to [30], complexes are formed in chitosan solutions in monocarboxylic acids by intermolecular crosslinking between the polymer macromolecules and the acid molecules as ion-ion-hydrogen contacts: an ionic bond between a protonated amino group of the polysaccharide and the oxygen in the hydroxyl group of a dissociated acid molecule and an $\mathrm{H}$-bond between the carbonyl oxygen of the acid and the $-\mathrm{OH}$ group at $\mathrm{C}_{6}$ in the poly- $D$-glucosamine. 


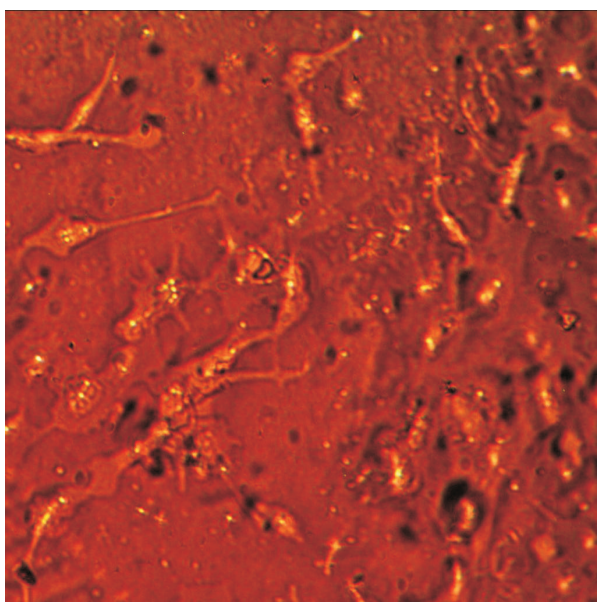

(a)

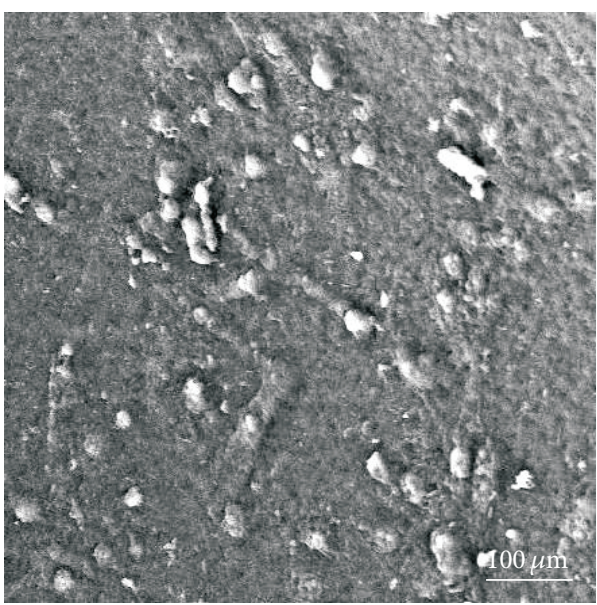

(c)

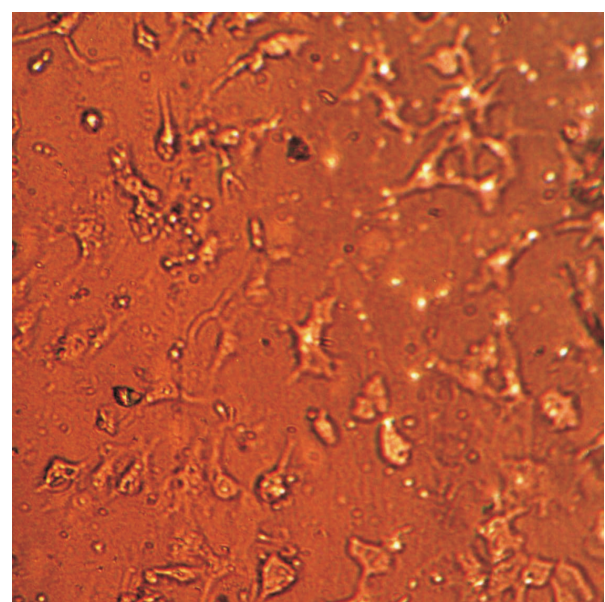

(b)

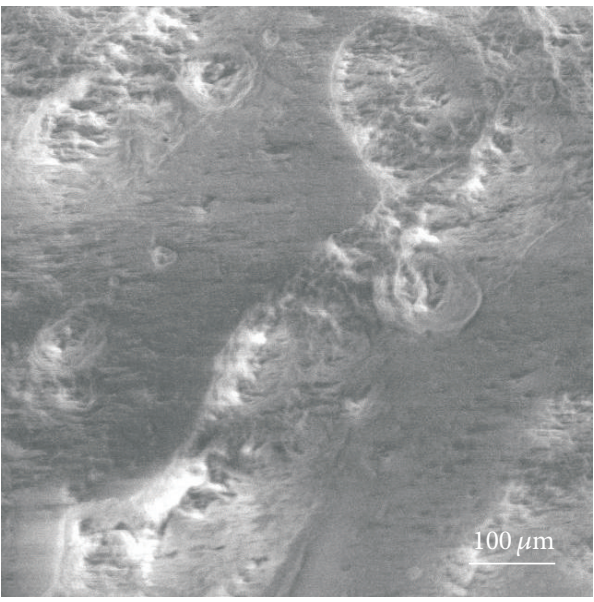

(d)

Figure 4: MA-104 cell culture after 4 days of cultivating ((a), (b)), light microscopy ( $\times 100$ scale); SEM images of a formalin-fixed cellular epitheliocyte culture after 7 days of cultivation $((\mathrm{c}),(\mathrm{d}))$ on the microtube surface obtained from the chitosan solution with the concentration $3.0 \mathrm{wt} . \%$ in a $1.5 \%$ aqueous solution of lactic acid by the wet ((a), (c)) and dry ((b), (d)) methods.

The size of such complexes in chitosan solution in tribasic citric acid is, perhaps, substantially larger as compared with chitosan solutions in monobasic lactic acid. Phase separation occurring while chitosan citrate with bulky citrate ions is converted to the polybasic form results in a more porous structure of the microtubes wall in comparison with similar processes in the case of chitosan lactate with substantially smaller lactate ions.

For the chitosan microtubes obtained by the wet process, stress-strain curves typical for viscoplastic materials are observed. As an example, the $\sigma=f(\varepsilon)$ dependence for the microtube sample obtained from the chitosan solution with $C_{\mathrm{CH}}=3.0 \mathrm{wt} . \%$ is presented (Figure 3 , curve 1). The stress-strain curves have two fragments, namely, an elasticdeformation fragment, whose value does not exceed $\sim 2-$ $2.5 \%$ and a fragment of plastic deformation which proceeds uniformly with no subsequent strain hardening and finishes with rupture of the sample onto two parts at one of the places of its fixing in the tensile machine's clips.
All microtube samples made by the wet method are characterized by low values of their tensile strength $\sigma=0.1-$ $0.7 \mathrm{MPa}$ but a rather high elongation at break $\varepsilon=21-45 \%$ (Table 1). $\sigma$ and $\varepsilon$ have their lowest values in the microtubes obtained from chitosan solutions with $C_{\mathrm{CH}}=2.5$ and $3.5 \mathrm{wt} . \%$ and the highest ones in the sample obtained from the chitosan solution with $C_{\mathrm{CH}}=3.0 \mathrm{wt}$.\%. Therefore, within the concentration range of the forming chitosan solutions, the $\sigma=f(C)$ and $\varepsilon=f(C)$ dependences are extreme.

A completely different behavior of the $\sigma=f(\varepsilon)$ curve is observed for the chitosan microtubes obtained by the dry process. Besides elastic deformation, these samples are characterized by their ability to show forced-elastic-deformation characteristic of viscoelastic materials (Figure 3, curves 2 and 3). Like the microtubes made by the wet process, the elastic-deformation of these samples is small $(\sim 2.5-3 \%)$. However, the value of $\sigma$ corresponding to the limit of proportionality (elastic deformation) is much higher in these samples and ranges within 6-12 MPa. Moreover, compared with the microtubes made by the wet method, the microtubes 


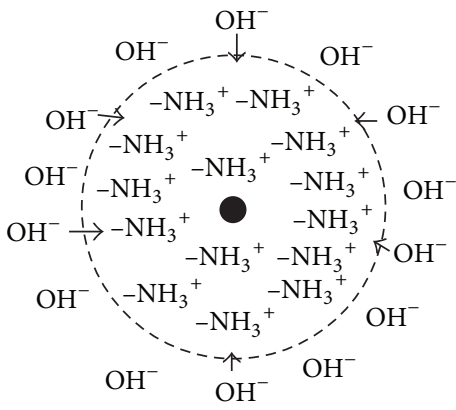

(a)

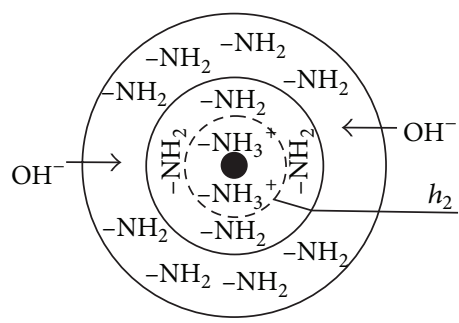

(c)

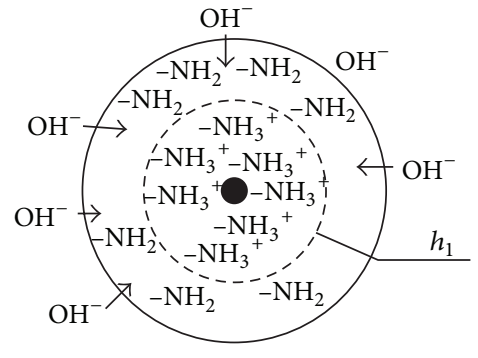

(b)

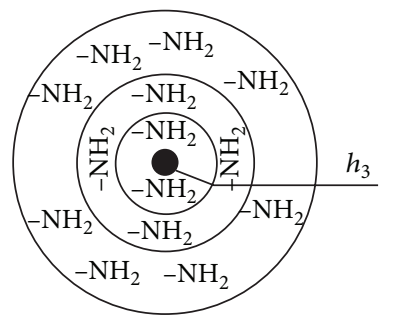

(d)

Figure 5: A schematic representation of the process of formation of a water-insoluble chitosan layer constituting the microtube's walls (a cross-section). See text for explanation.

obtained by the dry method deform under tensile tension less uniformly to form a neck due to the flow effect. This behavior of the $\sigma=f(\varepsilon)$ dependence is expressed most clearly for the microtube obtained from the solution with $C_{\mathrm{CH}}=3.0 \mathrm{wt}$. $\%$ (Figure 3, curve 2).

The dry method, as compared with the wet one, promotes the appearance of significantly stronger samples (Table 1). The sample obtained from the chitosan solution with $C_{\mathrm{CH}}=$ $3.0 \mathrm{wt} . \%$ has the highest strength. In comparison with the sample obtained from the chitosan solution of the same concentration but by the wet method, the value of $\sigma$ increases by more than 17 times. In the whole, the strength of microtubes produced according to the dry method is by 16-36 times higher than this value for microtubes obtained by the wet method. The concentration dependence $\sigma=f(C)$ of the tensile strength, like that for the wet-process microtubes, has a pronounced extremal character.

The microtubes obtained by the dry method, like those obtained by the wet method, are elastic. Within the used concentration range of the chitosan spinning solution, as $C_{\mathrm{CH}}$ increases, $\varepsilon$ rises from $8 \%$ up to $38.5 \%$.

All microtubes prepared by both molding processes are characterized by the almost uniform distribution of the wall thickness along the sample's length (Table 1). No clear correlation between the forming chitosan solution concentration and $d$ has been established.

Naturally, the thickness of the layer of the chitosan forming solution applied to the glass rod at the first stage of microtube formation was almost the same in all our experiments. However, the wall thickness of the wet-obtained finished microtubes is twice higher than that of those obtained by the dry method. These data show a more dense structure of the microtube walls molded by the dry method.
One can assume that the higher strength and smaller wall thickness of the microtubes made by the dry method are due to the presence of the stage of drying of the chitosan solution applied onto the glass rod at $45-50^{\circ} \mathrm{C}$ for $4-5 \mathrm{~h}$ (Figure 1). It appears that the polymeric structure is compacted at this stage, which continues during its subsequent treatment with a $\mathrm{NaOH}$ solution.

It should be noted that the elastic-deformation and strength characteristics of the microtube samples obtained from chitosan solutions of lactic acid by the dry method are not inferior to those of samples of xenopericardial biomaterials conventionally used in reconstructive surgery of the heart and blood vessels (Figure 3, curve 4) and samples of native blood vessels ( $\sigma=1.4-11.1 \mathrm{MPa}$ [31]).

Figure 4 shows the morphology of the cells cultured for 1 week on the microtube surface. Our experimental results show a high adhesion and a high proliferative activity of epithelial cells on the surface of the microtube substrates (Figures 4(a) and 4(b)).

\section{Conclusions}

Approaches to making chitosan microtubes by both wet and dry molding processes were developed. The designed methods are easy to perform and environmentally friendly. Our microtubes have no seams and represent an integral structure as a hollow cylinder. The mechanical properties of our microtubes made by the dry process correspond to those for xenopericardia, that is, the biomaterial successfully used in cardiac surgery for several decades. In vitro experiments show the high biocompatibility of our samples with epithelial-like cells. The obtained results allow us to 
consider our chitosan microtubes as promising analogues of biodegradable vascular prostheses.

\section{Conflict of Interests}

The authors declare that there is no conflict of interests regarding the publication of this paper.

\section{Acknowledgment}

The results of this work were obtained in the framework of implementation of the state task no. 4.1212.2014/K of the Russian Ministry of Education and Science.

\section{References}

[1] G. H. Naderi, D. Mehraban, S. M. Kazemeyni, S. R. Yahyazadeh, and A. H. Latif, "Polytetrafluoroethylene vascular graft as a rescuer of short renal vessels during kidney transplantation," Urology Journal, vol. 6, no. 1, pp. 47-49, 2009.

[2] S. Ravi and E. L. Chaikof, "Biomaterials for vascular tissue engineering," Regenerative Medicine, vol. 5, no. 1, pp. 107-120, 2010.

[3] K. M. J. Chan, S. Rahman-Haley, T. K. Mittal, J. A. Gavino, and G. D. Dreyfus, "Truly stentless autologous pericardial aortic valve replacement: an alternative to standard aortic valve replacement," Journal of Thoracic and Cardiovascular Surgery, vol. 141, no. 1, pp. 276-283, 2011.

[4] Internet resource, http://www.1tv.ru/news/health/246940.

[5] N. Bölgen, Y. Z. Menceloğlu, K. Acatay, I. Vargel, and E. Pişkin, "In vitro and in vivo degradation of non-woven materials made of poly( $\varepsilon$-caprolactone) nanofibers prepared by electrospinning under different conditions," Journal of Biomaterials Science, Polymer Edition, vol. 16, no. 12, pp. 1537-1555, 2005.

[6] R. T. Tran, W. M. Choy, H. Cao et al., "Fabrication and characterization of biomimetic multichanneled crosslinked-urethanedoped polyester tissue engineered nerve guides," Journal of Biomedical Materials Research A, vol. 102, no. 8, pp. 2793-2804, 2014.

[7] C. Y. Xu, R. Inai, M. Kotaki, and S. Ramakrishna, "Aligned biodegradable nanofibrous structure: a potential scaffold for blood vessel engineering," Biomaterials, vol. 25, no. 5, pp. 877886, 2004.

[8] T. B. Bini, S. Gao, T. C. Tan et al., "Electrospun poly(Llactide-co-glycolide) biodegradable polymer nanofibre tubes for peripheral nerve regeneration," Nanotechnology, vol. 15, no. 11, pp. 1459-1464, 2004.

[9] M. J. Smith, M. J. McClure, S. A. Sell et al., "Suture-reinforced electrospun polydioxanone-elastin small-diameter tubes for use in vascular tissue engineering: a feasibility study," Acta Biomaterialia, vol. 4, no. 1, pp. 58-66, 2008.

[10] S. Panseri, C. Cunha, J. Lowery et al., "Electrospun micro- and nanofiber tubes for functional nervous regeneration in sciatic nerve transections," BMC Biotechnology, vol. 8, article 39, 2008.

[11] C. M. Vaz, S. van Tuijl, C. V. C. Bouten, and F. P. T. Baaijens, "Design of scaffolds for blood vessel tissue engineering using a multi-layering electrospinning technique," Acta Biomaterialia, vol. 1, no. 5, pp. 575-582, 2005.

[12] X. Hu, H. Shen, F. Yang, J. Bei, and S. Wang, "Preparation and cell affinity of microtubular orientation-structured
PLGA(70/30) blood vessel scaffold," Biomaterials, vol. 29, no. 21, pp. 3128-3136, 2008.

[13] J. Yang, D. Motlagh, A. R. Webb, and G. A. Ameer, "Novel biphasic elastomeric scaffold for small-diameter blood vessel tissue engineering," Tissue Engineering, vol. 11, no. 11-12, pp. 1876-1886, 2005.

[14] A. A. Barros, A. R. C. Duarte, R. A. Pires, A. Lima, J. F. Mano, and R. L. Reis, "Tailor made degradable ureteral stents from natural origin polysaccharides," in Proceedings of the Materials 10th Conference on Supercritical Fluids and Their Applications, pp. 1-6, 2013.

[15] S. Lepidi, F. Grego, V. Vindigni et al., "Hyaluronan biodegradable scaffold for small-caliber artery grafting: preliminary results in an animal model," European Journal of Vascular and Endovascular Surgery, vol. 32, no. 4, pp. 411-417, 2006.

[16] X. Kong, B. Han, H. Wang, H. Li, W. Xu, and W. Liu, "Mechanical properties of biodegradable small-diameter chitosan artificial vascular prosthesis," Journal of Biomedical Materials Research A, vol. 100, no. 8, pp. 1938-1945, 2012.

[17] C. Zhu, D. Fan, Z. Duan et al., "Initial investigation of novel human-like collagen/chitosan scaffold for vascular tissue engineering," Journal of Biomedical Materials Research A, vol. 89, no. 3, pp. 829-840, 2009.

[18] I. Y. Kim, S. J. Seo, H. S. Moon et al., "Chitosan and its derivatives for tissue engineering applications," Biotechnology Advances, vol. 26, no. 1, pp. 1-21, 2008.

[19] I. Matsumoto, M. Kaneko, M. Oda, and G. Watanabe, "Repair of intra-thoracic autonomic nerves using chitosan tubes," Interactive Cardiovascular and Thoracic Surgery, vol. 10, no. 4, pp. 498-501, 2010.

[20] S. Wang, Y. Zhang, H. Wang, G. Yin, and Z. Dong, "Fabrication and properties of the electrospun polylactide/silk fibroingelatin composite tubular scaffold," Biomacromolecules, vol. 10, no. 8, pp. 2240-2244, 2009.

[21] T. Freier, R. Montenegro, H. S. Koh, and M. S. Shoichet, "Chitinbased tubes for tissue engineering in the nervous system," Biomaterials, vol. 26, no. 22, pp. 4624-4632, 2005.

[22] X. F. Zhang, W. L. Cao, Y. D. Gong, Y. Gao, and J. M. Li, “A method for the preparation of porous chitosan tube," China Patent no ZL 02 149086.4. 2005.

[23] L. Zhang, Q. Ao, A. Wang et al., "A sandwich tubular scaffold derived from chitosan for blood vessel tissue engineering," Journal of Biomedical Materials Research A, vol. 77, no. 2, pp. 277-284, 2006.

[24] W. Wang, S. Itoh, A. Matsuda et al., "Influences of mechanical properties and permeability on chitosan nano/microfiber mesh tubes as a scaffold for nerve regeneration," Journal of Biomedical Materials Research A, vol. 84, no. 2, pp. 557-566, 2008.

[25] L. S. Kuchanskaya, N. O. Gegel, and A. B. Shipovskaya, "Preparation of chitosan-based microtubes," in 11th International Conference on Modern Perspectives in the Study of Chitin and Chitosan Materials, pp. 59-63, Murmansk, Russia, 2012.

[26] T. S. Babicheva and A. B. Shipovskaya, "Preparation and study of the physical and mechanical properties of chitosan microtubes," in Proceedings of the Modern Problems of Theoretical and Experimental Chemistry. Intercollege. Sat Scientific. IX All Works. Conference Young. Science with International. Participation, pp. 138-140, Saratov, Russia, 2013.

[27] Y. I. Afanasiev, N. A. Yurina, E. F. Kotovskij et al., Histology, Embryology, Cytology: A Textbook, GEOTAR Media, Moscow, Russia, 6th edition, 2012. 
[28] H. Storrie and S. I. Stupp, "Cellular response to zinc-containing organoapatite: an in vitro study of proliferation, alkaline phosphatase activity and biomineralization," Biomaterials, vol. 26, no. 27, pp. 5492-5499, 2005.

[29] Z. Wang, Q. Hu, and L. Ca, "Chitin fiber and chitosan 3D composite rods," International Journal Polymer Science, vol. 2010, Article ID 369759, 7 pages, 2010.

[30] Q.-X. Li, B.-Z. Song, Z.-Q. Yang, and H.-L. Fan, "Electrolytic conductivity behaviors and solution conformations of chitosan in different acid solutions," Carbohydrate Polymers, vol. 63, no. 2, pp. 272-282, 2006.

[31] V. A. Kumar, J. M. Caves, C. A. Haller et al., "Acellular vascular grafts generated from collagen and elastin analogs," Acta Biomaterialia, vol. 9, no. 9, pp. 8067-8074, 2013. 

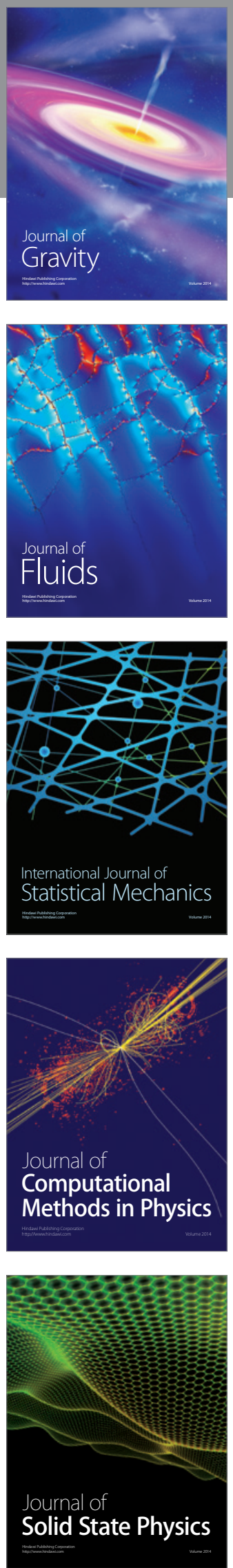

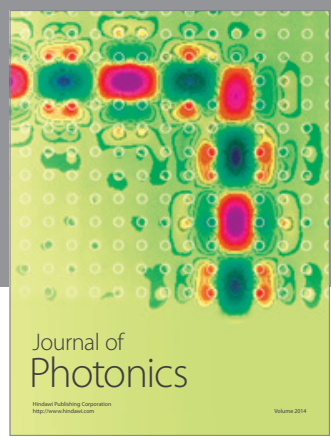

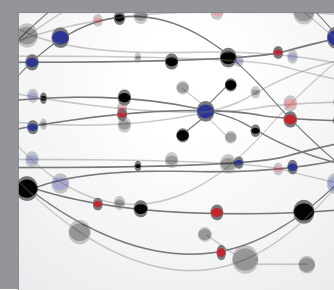

The Scientific World Journal

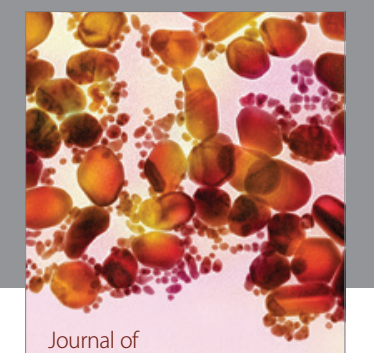

Soft Matter
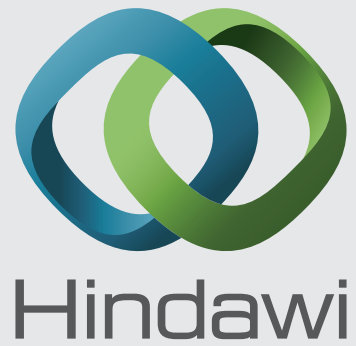

Submit your manuscripts at

http://www.hindawi.com
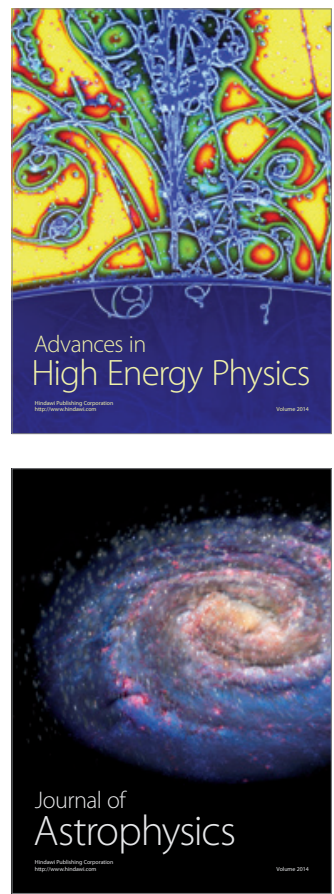
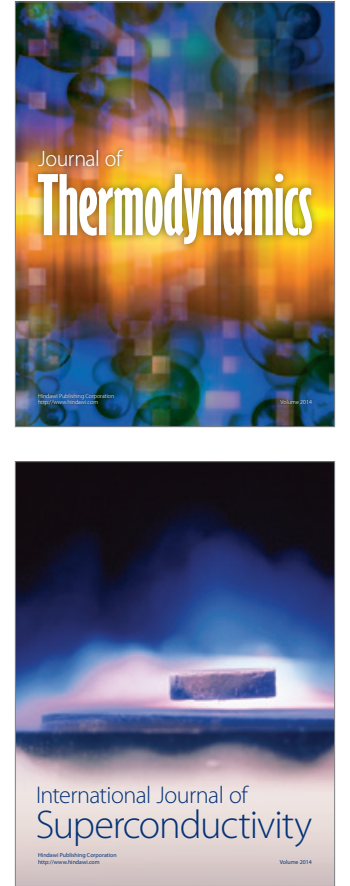
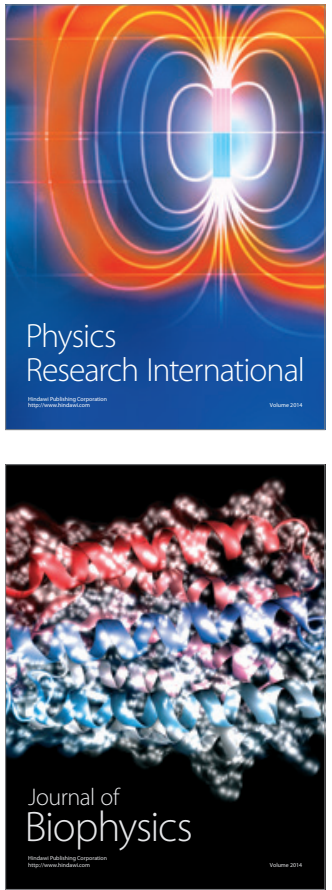
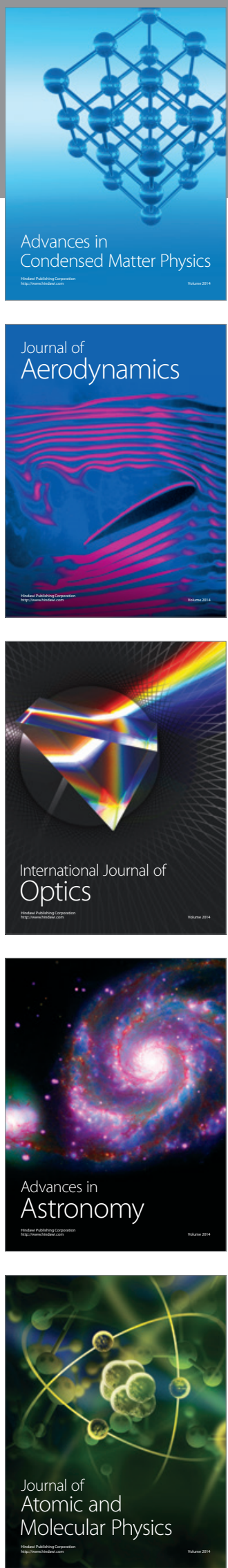